\title{
Modulation of the Immune System by Human Rhinoviruses
}

\author{
Stefanie Kirchberger Otto Majdic Johannes Stöckl \\ Institute of Immunology, Medical University of Vienna, Vienna, Austria
}

\section{Key Words}

Human rhinovirus - Immune evasion - Dendritic cells .

Monocytes $\cdot \mathrm{IL}-10 \cdot \mathrm{B} 7-\mathrm{H} 1 \cdot$ Sialoadhesin

\begin{abstract}
Human rhinoviruses (HRV) are the major cause of the common cold, one of the most frequent infectious diseases in humans. Though HRV infections of the upper respiratory tract are usually rather harmless, there is increasing evidence that HRV sets the stage for more dangerous pathogens, elicits asthmatic exacerbations, severe diseases in the lower respiratory tract and even autoimmunity. The pathogenic mechanisms of HRV infections leading to such complications are still poorly understood. It is a common strategy of pathogens to manipulate our immune system in order to evade an efficient immune response. A major characteristic of $\mathrm{HRV}$ is a high degree of species specificity. Thus, analyzing the potential immune evasion mechanisms used by HRV will be helpful for a better understanding of the pathogenesis of the common cold and may contribute to a better understanding of the human immune system as well. In this review we want to illuminate what is known about potential immune escape mechanisms used by HRV and discuss how such disturbances might lead to a suppressed and dysregulated immune competence in man.
\end{abstract}

Copyright $\odot 2007$ S. Karger AG, Basel

\section{Introduction}

For millions of years, viruses have studied cell biology and immunology the hard way, to acquire and defend an ecological niche for replication [1]. The success of a virus is directly dependent on its ability to mount an effective anti-immune response within the infected host. As a consequence, successful viruses have developed a broad range of mechanisms to modulate, manipulate or shut off key functions of the host immune system. Many cornerstones of immunity have been illuminated by analyzing the underlying mechanisms of effective immune escape strategies of viruses. This 'anti-immunology' covers an incredible repertoire of different strategies and is best defined for large DNA viruses such as herpesvirus or cytomegalovirus $[2,3]$. It is amazing that such large DNA viruses utilize up to $50 \%$ of their genome for proteins specialized to interfere with the host immune system including a plethora of cytokine homologs, mimics or inhibitors of innate and acquired immune responses to facilitate their replication in hosts with complex and effective immune systems [1].

Owing to the low fidelity of RNA polymerase, the genome size of RNA viruses is limited and usually small [4]. Although this confers the advantage of a fast replication cycle and the ability to use mutation to escape immune control, there is little room in the genome to allow im-

\section{KARGER \\ Fax +4161306 1234 \\ E-Mail karger@karger.ch}

www.karger.com (c) 2007 S. Karger AG, Basel

$1018-2438 / 07 / 1421-0001 \$ 23.50 / 0$

Accessible online at:

www.karger.com/iaa
Correspondence to: Dr. Johannes Stöckl

Institute of Immunology, Medical University of Vienna

Borschkegasse 8a, AT-1090 Vienna (Austria)

Tel. +43 14277 64951, Fax +431 42779649

E-Mail Johannes.Stoeckl@meduniwien.ac.at 
mune defenses to be encoded by individual genes. Human rhinoviruses (HRV) are such small RNA viruses and are nevertheless one of the most successful pathogens in humans [5-7]. It is tempting to postulate that rapidly evolving HRV have achieved the same goals as large DNA viruses, notwithstanding their significantly reduced coding capacity, by exploiting some of the cellular mechanisms that the host may have evolved for the regulation of immune responses.

\section{Rhinoviruses}

HRV are members of the Picornaviridae [7]. So far, 102 serotypes of HRV have been identified and are divided into two groups depending on receptor utilization. More than $90 \%$ belong to the major group family of HRV and bind to human intercellular adhesion molecule-1 (ICAM-1), whereas low-density lipoprotein and related proteins are receptors for the minor group $\operatorname{HRV}[8,9]$. HRV are non-enveloped viruses with an icosahedral capsid enclosing a single-stranded, positive-sense RNA genome that is translated after cell entry [10]. The viral polyprotein is divided into a $\mathrm{P} 1$ region, which encodes the capsid proteins VP1, VP2, VP3, and VP4, and the P2 and $\mathrm{P} 3$ regions, which include proteins $2 \mathrm{APro}, 2 \mathrm{~B}, 2 \mathrm{C}, 3 \mathrm{~A}, 3 \mathrm{~B}$ (VPg), 3CPro, and 3DPol. Viral proteases cleave the polyprotein into the component viral proteins, with the cleavage end products, as well as some of the precursors, having independent functions. Importantly, for none of these proteins have such 'anti-immunological' functions been described.

An HRV infection normally starts by self-inoculation with the virus by hand into the nose or into the eyes, from where the virus comes via the lacrimal duct into the nose [11]. In the adenoid area of the nose the viruses dock to epithelial cells via specific cellular receptors. Receptor binding of major group viruses leads to conformational changes of the capsid, which facilitate together with a low $\mathrm{pH}$ uncoating within endosomes and release of viral RNA $[12,13]$. While the host cell protein synthesis is shut off by the cleavage of the CAP-binding complex through viral protease $2 \mathrm{a}$, the viral RNA can still be translated using an internal ribosome entry site [14]. Assembled mature virions are released from the cell after $8-10 \mathrm{~h}$ by cell lysis of epithelial cells. Although HRV primarily infect the ciliated epithelial cells of the upper respiratory tract, there is increasing evidence that HRV can also replicate in the lower respiratory tract. Histological examinations of HRV-infected nasal epithelium demonstrated no obvious changes in the morphology or integrity of the nasal epithelium. HRV is found to replicate only in a small number of mucosal cells (about 10\%) and induces little, if any, cytopathic effects [11].

HRV infection of primary epithelial cells and epithelial cell lines is accompanied by a release of inflammatory mediators in vivo and in vitro. In particular pro-inflammatory cytokines including IL-1 $\beta$, TNF, IL-8, IL-6 and IL-11, and the vasoactive peptides bradykinin and lysyl bradykinin $[15,16]$. In addition, chemokines such as Rantes, MCP-1 and IP-10 as well as the angiogenic factor VEGF have been found in nasal secretions of patients with colds [16-19]. As a result, it is now considered that common cold symptoms result from an inflammatory 'cytokine disease' of the host in response to the virus and not from the virus itself.

Attracted by these mediators, inflammatory leukocytes, granulocytes, dendritic cells (DC) and monocytes are found to migrate to the site of HRV infection [20]. Especially neutrophil infiltration into the submucosa and epithelium was observed during the common cold and may be caused by IL- 8 , which is a potent chemoattractant and mediator for the invasion of neutrophils. Teran et al. [21] found a direct relation between neutrophils and myeloperoxidase concentrations in nasal aspirates and the severity of upper respiratory tract symptoms. The increased presence of neutrophils has been implicated to contribute to asthmatic exacerbations. In contrast to allergen-provoked asthma where mainly eosinophils are attracted, neutrophils are the predominant inflammatory cells during virally induced asthma exacerbations [22]. However, it was also reported that eosinophils are increased in bronchial epithelium in biopsies taken from normal and asthmatic persons following experimental HRV infection [23]. In addition, neutrophilia did not differentiate between the response to virus in a control population or in patients with asthma with an exacerbation during the acute infection. This observation suggests that factors other than, or more likely in addition to, neutrophils are necessary for an asthma exacerbation to occur with a respiratory virus infection.

In summary, it appears that a small number of HRVinfected epithelial cells release a variety of inflammatory factors. These soluble mediators are thought to orchestrate proliferation, chemotaxis, and activation of inflammatory cells, resulting in an amplification of the inflammatory process. 


\section{The Immune Response against HRV}

Several studies demonstrated that an intact immune system is required to control HRV infections. This is particularly clear from studies with patients with a suppressed immune system, where HRV infections can be life-threatening and are a major cause of morbidity [6].

A typical antiviral immune response is a combination of innate and adaptive immunity. Type-I interferons (IFN) are known as the prototypic and early mediators of an innate antiviral immune response [24]. IFN is detectable in nasal secretions of one third of infected volunteers and it is usually present 1 or 2 days after the peak virus titer [5]. However, it is detected only in those volunteers with the highest titers of virus and most severe illness. For this reason, many believe other recovery mechanisms may be more important. Nevertheless, administration of IFN before challenge with HRV can prevent or reduce the intensity of infection. A recent paper indicated an important link between IFN and the increased susceptibility of asthma patients to HRV. Wark et al. [25] showed that bronchial epithelial cells of asthmatic subjects have an impaired apoptotic response to HRV infections and increased viral replication due to a profound impairment of virus-induced IFN expression. Substitution of IFN induced apoptosis and reduced replication. Thus, IFN may play a role in recovery from HRV infections as well as in the prevention of virus-induced asthmatic exacerbations.

Neutralizing antibodies to HRV develop after infection in serum and secretions of the majority of persons and are considered as the most important antiviral effector mechanism of our adaptive immune system [5]. Among infected volunteers, detectable serum antibody usually developed between 1 and 2 weeks after inoculation, but in initially antibody-free persons, it might first be detected after 3 weeks. Maximum levels of specific antibodies are reached after 5 weeks and can persist for at least 1 year. However, as neutralizing antibodies appear relatively late, recovery from illness, which usually occurs at 7-10 days, must be a result of other components of the immune response. This concept is supported by a seemingly normal recovery exhibited by persons with isolated IgA deficiency and by those with hypogammaglobinemia [26]. Nevertheless, local IgA and also IgG antibodies, passing from the vasculature may contribute to viral clearance in otherwise healthy persons. However, the large number of serotypes means repeated infection with HRV, to which an individual may lack appropriate antibodies, is common. Moreover, antibody production in natural HRV infections occurs on an average only in $50 \%$ of patients [5].

In contrast to the specific antibody response, $\mathrm{T}$ cells show serotype cross-reactivity. Gern et al. [27] isolated virus-specific CD4+ T-cell clones and showed that cells from most persons are activated by a number of serotypes, indicating shared epitopes by different HRV. T cells from tonsils of infected children also responded to multiple serotypes; responses were by $\mathrm{CD} 4+$ cells and a Th1-type cytokine profile was dominating [28]. Surprisingly, Handzel et al. [29] reported that eosinophils might act as the antigen-presenting cells, which activate HRVspecific $T$ cells. Today, no publications are available about an involvement of CD8+ T cells in the immune responses against HRV. This is amazing, since $\mathrm{CD} 8+$ cytotoxic $\mathrm{T}$ cells usually play a central role in the fight against noncytopathic viruses.

Taken together, the immune response against HRV as well as the recovery from HRV infection is still incompletely understood and enigmatic.

\section{Immune Evasion Strategies of HRV}

Antigenic variation and rapid replication are critical immune evasion mechanisms of small RNA viruses $[1,2]$. Therefore the large numbers of serotypes combined with a high mutation rate might be part of the success of HRV infections. However, the intrinsic antigen variations of HRV do not explain why, despite recruitment of invading leukocytes, appropriate immune responses appear to be hindered or dysregulated in the respiratory tract upon HRV infection, leading to a well-documented disposition to bacterial infections leading to sinusitis, otitis media, bronchitis, and pneumonia as well as asthmatic exacerbations and autoimmunity [6,30-34]. This property cannot be attributed to cytopathic effects because several studies have clearly demonstrated that HRV bind to but do not infect or damage leukocytes $[35,36]$. In the next chapters we want to discuss the cellular and molecular mechanisms through which HRV blunts our immune system.

\section{Interference of HRV with the Type I IFN Pathway}

Many viruses express mechanisms to counteract the host response by targeting the antiviral power of IFNs. IFNs elicit antiviral activity by the induction of different already well-studied interferon-stimulated genes (ISG) applying different modes of action [24]. Examples for 
such ISG are the double-stranded RNA-activated kinase PKR, which inhibits mRNA translation and transcriptional events; the enzymes 2 ', $5^{\prime}$-oligoadenylate synthetase and RNase L, which are involved in mRNA degradation, and the Mx proteins, which are GTPases. These GTPases are expressed in two distinct forms in humans, as $\mathrm{MxA}$ and $\mathrm{MxB}$, whereas only MxA has antiviral activity. It can act against a number of different viruses including also Picornaviridae. Interaction of MxA with viral nucleocapsids followed by misdirection of viral components was described as a possible antiviral mechanism [37]. Although MxA was found to be not systemically upregulated in common cold patients, it could be elevated locally in the nasal mucosa [38].

The regulation of type-I IFN synthesis is well characterized and requires the participation of several transcription factor complexes, such as NF- $\mathrm{B}, \mathrm{ATF} / \mathrm{JUN}$ and, in particular, IFN regulatory factors (IRFs). Since many viruses are known to target activation of IRF-3 to inhibit the induction of IFN- $\beta$, Peng et al. [39] have recently analyzed the status of IRF-3 in HRV-infected epithelial cell lines. IRF-3 was translocated to the nucleus and phosphorylated in cells infected with HRV14, a major group HRV. Despite this apparent activation, very little homodimerization of IRF-3 was evident following infection. Similar results in A549 lung alveolar epithelial cells demonstrated the biological relevance of these findings to HRV14 pathogenesis. In addition, prior infection of cells with HRV14 prevented the induction of IFN- $\beta$ mRNA following treatment with double-stranded RNA, indicating that HRV14 may encode an activity or induces a negative signal that specifically inhibits this innate host defense pathway. The exact mechanisms by which HRV14 inhibits IRF-3 homodimerization remain to be delineated.

\section{Modulation of Leukocyte Interactions}

The selection of an immunologically relevant cellular receptor is an efficient anti-immune response strategy used by several viruses. This is probably best characterized for measles viruses, which abuse binding to CD46 to blunt immunity. The vast majority of HRV uses the cell surface receptor ICAM-1 (CD54) to bind to and infect epithelial cells. ICAM-1 is a member of the immunoglobulin (Ig) superfamily and is important in adhesion of leukocytes and endothelial leukocyte migration through binding to its cellular ligands LFA-1 (CD11a/CD18) and Mac-1 (CD11b/CD18) [40]. Mice deficient in ICAM-1 fail to recruit leukocytes to inflamed tissues [41]. ICAM-1 is also an essential co-stimulatory receptor on antigen-presenting cells and ICAM-1-deficient APC have a strongly impaired capacity to induce T-cell responses $[42,43]$. Thus, HRV can directly interact with leukocytes and since the binding site of HRV on ICAM-1 overlaps with the binding site of LFA-1, it is intriguing that binding of HRV to leukocytes might have profound functional consequences. Piela-Smith et al. [44] could indeed demonstrate that ICAM-1 expressed on the surface of human fibroblasts functions as a receptor for HRV as well as a receptor for LFA-1-bearing human T lymphocytes, which could be inhibited by an antibody to ICAM-1. Interestingly, binding of HRV to fibroblasts inhibited the subsequent adhesion of $\mathrm{T}$ cells.

Similarly, Gern et al. [45] found that HRV16, a major group HRV, significantly inhibited T-cell proliferation induced by antigen but not proliferation secondary to mitogens, IL-2, or an irradiated allogeneic T-cell line. Noninfectious (UV-irradiated) HRV had similar effects. Inhibition of T-cell proliferation was dependent on HRV binding to ICAM-1 on monocytes, indicating that the virus interferes with lymphocyte activation indirectly through effects on antigen-presenting cells. In addition, HRV inhibited T-cell cytotoxic responses but not NK cell activity.

Recent studies have clearly demonstrated that ICAM-1 is more than a molecular glue [36, 46-48]. Engagement of ICAM-1 with certain $m$ Abs or its natural ligands fibrinogen was described to trigger intracellular signaling in various cell types. For other prominent adhesion molecules including ICAM-3 (CD50), signaling events could be observed, leading to the activation of other adhesion molecules and thereby reinforcing the strength of intercellular contact formation.

HRV is a natural ligand for ICAM-1 with special binding and consequently signaling features. One HRV capsid has 60 potential binding sites located in depressions of the viral protein coat, the so-called canyons [49]. The binding of HRV to ICAM-1 was found to be biphasic. It starts with a slow, low-affinity interaction step, which causes a conformational change in the canyons, which facilitates HRV/ICAM-1 interactions with a relatively high affinity. These structural changes in the canyons subsequently favor binding of additional ICAM-1 molecules and it is assumed that numerous ICAM-1 molecules get located around the initial binding site of HRV. Due to the multivalency of binding sites on HRV particles, this allows high avidity interactions with their receptors. Therefore, HRV can be considered to resemble a perfect cross-link- 
ing reagent of ICAM-1 molecules, which may trigger proadhesive effects as well.

We have recently investigated whether HRV binding to ICAM-1 might influence the adhesiveness of leukocytes [50]. We found that engagement of ICAM-1 with HRV14 on human mononuclear phagocytes induces homotypic clustering of monocytes and monocyte-derived DC, but not in lymphocytes, which was found to critically involve homophilic PECAM-1 interactions and is mediated via LFA-1/ICAM-3.

Three pieces of evidence demonstrated that engagement of ICAM-1 by HRV is required to elicit the evoked adhesiveness of monocytes. Inhibition of binding of HRV14 to monocytes with the specific inhibitor WIN 52035-2 or the mAb 17-1A, which block HRV binding to ICAM-1, abolished the HRV induced clustering. Secondly, heat-inactivated HRV14, which is unable to bind to ICAM-1, failed to induce aggregation of monocytes. Thirdly, induction of monocyte or DC aggregation was not observed with the minor group HRV2, which interacts with cells via LDL-receptor- and/or LDL-receptorrelated proteins. Cell surface expression of ICAM-1 on monocytes was reduced upon HRV14 interaction. This is likely to be primarily due to receptor usage of HRV14. In addition, this enhanced cellular adhesiveness was also observed with UV-inactivated HRV preparations that can still bind to ICAM-1 but are not infectious.

Cross-linking of ICAM-1 by antibodies was shown to activate the ERK-1/2 and JNK-1/2 signaling pathways. Binding of HRV to monocytes also induces ERK and JNK phosphorylation [Kirchberger et al., unpubl. data]. It remains to be determined whether this signaling route is responsible for the enhanced adhesiveness of monocytes upon HRV binding.

The amount of HRV particles required to induce this effect was low. Thus it can be anticipated that mononuclear phagocytes, which migrate to the site of HRV infection, come into contact with sufficient viruses to acquire this enhanced adhesive state. What might be the relevance of enhanced phagocyte adhesiveness? Mononuclear phagocytes with such an enhanced adhesive behavior might be retained longer at the infected site than usual. Moreover, a delayed emigration of APC to local lymphoid tissues and lymph nodes might cause a delayed immune response to virus. This possibility could help to explain why it usually takes 3-5 weeks until neutralizing mAbs against HRV are detectable [5].

Modulation of the Immune System by Human Rhinoviruses

\section{Modulation of Cytokine Production in Monocytes}

Mononuclear phagocytes were considered for a long time as a primitive cell system that is involved in unspecific, first-line defense mechanisms [51, 52]. The picture changed when it became clear in the mid-1990s that phagocytes resemble precursor cells of DC, the key players in the regulation and induction of specific immunity. This progress also caused a revision of the concept that macrophages and DC may originate from different precursor cells and was replaced by a more flexible model which explains the heterogeneity of this cell system by the influence of microenvironmental factors including specific cytokines as well as pathogen-associated signals. The capacity of mature CD14+ monocytes to further differentiate in either one or the other 'functional direction' was called 'polarized' monocyte differentiation according to the Th1, 2, 3 nomenclature used for T cells.

Data from a recent study suggest that HRV may polarize monocyte differentiation towards a 'Th-3 phenotype' [36]. In this study, we demonstrated that HRV14 efficiently inhibit antigen-specific T-cell responses. These inhibitory effects were found to result from a particular spectrum of cytokines released by mononuclear phagocytes upon HRV14 interaction. Most prominent among them is IL-10, a well-established immunosuppressive cytokine, which is strongly induced by HRV14. In contrast, major pro-inflammatory cytokines such as TNF- $\alpha$ and IL-1 $\beta$ as well as the Th1 activator IL-12 are not or only minimally induced and IL-12 production induced by IFN- $\gamma /$ LPS was significantly inhibited in monocytes after HRV14 interaction. On the basis of these observations it is tempting to speculate that the particular cytokine profile induced by HRV in mononuclear phagocytes might have adverse effects on local immunity in areas of HRV infection. Such reduced local immunocompetence may predispose affected individuals to secondary infections and could possibly explain the frequently observed occurrence of sinusitis, otitis media, bronchitis, and pneumonia in $\mathrm{HRV}$-infected persons.

While direct interference of HRV binding to ICAM-1 on monocytes and consequently inhibition of LFA-1 binding may contribute to a reduction of T-cell reactivity, it cannot account for the inhibitory effects of HRV observed by us. In particular, HRV14 did not inhibit allogeneic T-cell responses induced by EBV-transformed lymphoblastoid B cell lines (B-LCL), where ICAM-1/LFA-1 interactions are also critically involved [36]. Rather, the induction of a soluble factor by HRV14 in monocytes seems to be primarily responsible for the inhibition of the

Int Arch Allergy Immunol 2007;142:1-10 
antigen-specific T-cell response. Data presented in this study demonstrate that this factor is IL-10. Firstly, addition of supernatant from HRV14-treated monocytes only inhibited the allo-stimulatory capacity of monocytes and md-DC whereas B-LCL were not affected, which is typical for IL-10. Secondly, monocytes stimulated with HRV14 were found to produce biologically relevant amounts of IL-10. Thirdly, neutralization of IL-10 with a specific antibody reverted the blocking effects of HRV on T-cell stimulation.

The mechanism of inhibition of antigen-specific, cellular immune responses by IL-10 is well defined. Most relevant in this respect is the massive downregulation of MHC class II molecule expression in the presence of IL-10 [53]. Accordingly, HRV14-induced downregulation of MHC class II molecules was inhibited in the presence of anti-IL-10 antibodies. In contrast to MHC class II, MHC class I expression on monocytes is only slightly affected by IL-10 and responsiveness of activated CD8+ $\mathrm{T}$ cells is even enhanced by IL-10. It has also been demonstrated that IL-10 reduces CD80 and CD86 co-stimulatory molecule expression and inhibits IL-12 production. Upon HRV14 treatment of monocytes, we have also observed downregulation of CD86 expression as well as an inhibition of IL-12 production. However, inhibition of IL-12 production induced by HRV14 was only partially reversed in the presence of neutralizing anti-IL-10 antibodies, indicating that an IL-10-independent mechanism is also critically involved, which is reminiscent to what has been found with measles viruses [54]. Also, in contrast to IL-10 effects, CD80 as well as CD40 expression was upregulated in HRV-treated monocytes, which was also reported in a study from another group [55]. Thus, the immunophenotype of HRV14-treated monocytes differs in some aspects from that reported for IL-10 stimulation and not all functional effects of HRV14 may be due to induction of IL-10 production.

IL-10 is one of the key factors that regulate inflammatory responses [56]. The anti-inflammatory action of IL-10 is primarily achieved by inhibition of the production of pro-inflammatory cytokines such as IL-1, IL-6 and TNF- $\alpha$. The crucial role of IL-10 in controlling inflammatory responses is best illustrated in IL-10 knockout mice which suffer from chronic inflammatory bowel disease and in studies where injection of IL-10 rescued mice from LPS-induced toxic shock $[57,58]$. Common cold symptoms are considered to be caused by pro-inflammatory cytokines produced in the nasal mucosa [5]. In the course of the infection, however, major group HRV may induce the production of IL-10 in infiltrating mono- cytes. This may result, together with IL-1ra, in a decrease of the local inflammatory reaction and in the inhibition of cellular immune responses.

The utilization of IL-10 for immunomodulation is a common strategy of viruses. Members of the herpesvirus and parapoxvirus families have evolved their own IL-10 homologs, whereas other viruses have developed strategies to induce cellular IL-10 [1]. The exact molecular mechanisms by which viruses such as respiratory syncytial virus, parainfluenza virus or HRV cause increased secretion of IL-10 by monocytes and macrophages have not been determined. However, it is apparent that induction of cellular IL-10 by viruses that target macrophages leads to suppression of the localized - and perhaps generalized - inflammatory response. This immunosuppression is likely to be beneficial to the virus in establishing an infection. Understanding how microorganisms initiate macrophage production of IL-10 and regulate IL-10 transcription and translation could reveal important targets for therapeutic intervention in a wide variety of diseases.

Amazingly, IL-10 might be the 'missing link' to explain the linkage between a viral respiratory infection and the provocation of an asthma exacerbation. A recent study by Grissell et al. [59] points to IL-10 as an indicator of HRV-provoked asthma, both in children and adults. The observations by Grissell et al. add critical new information as we try to understand the mystery of how an innocuous respiratory infection, like the common cold produced by HRV, can have such devastating effects in some patients with asthma.

\section{Immune Evasion by Targeting DC}

DC, the most potent of the professional antigen-presenting cells, play a central role in the generation of primary T-cell responses and the maintenance of immunity. Because of their importance in initiating antiviral immune responses, DC are ideal targets for viral immune evasion strategies $[2,60]$. Several viruses are known to modulate DC function and thus impair antiviral T-cell responses. The result can be a transient or prolonged suppression of the immune response, often associated with secondary microbial infections or the initiation of a persistent infection, both of which represent serious medical problems. However, the mechanism(s) by which viruses induce dysfunction in DC is not fully understood.

Viruses can interfere at several steps of DC-induced immunity including generation, survival, migration and maturation of DC, processing/presentation of antigens 
by DC and activation of T-cell responses by DC $[2,60]$. A particularly efficient trick of viruses, e.g. vaccinia virus, measles, HIV or CMV, is the targeting of the co-stimulatory function of DC. Thereby the virus is able to prevent pathogen-specific immunity (i.e. ignorance) and, even more effective, is able to induce pathogen-specific tolerance, when DC present antigens in the absence of costimulatory signals. We have recently demonstrated that upregulation of inhibitory accessory molecules, i.e. B7H1 (CD274) and Sn (sialoadhesin, Siglec-1, CD169), on DC represents another efficient viral strategy to turn off antigen-specific immune responses [61].

We discovered this novel mechanism of virus-induced immunosuppression by investigating the impact of HRV14 on DC function. Since monocytes are the precursor cells of our DC, we expected that IL-10 production induced upon co-culture of DC with HRV14 was involved in this process. Yet, although small amounts of IL-10 are produced by R-DC, neutralizing antibodies against IL-10 failed to reverse the inhibitory effect of HRV14. In addition, transfer experiments with the supernatant of R-DC cultures revealed that soluble inhibitory factors may not play an essential role. Thus, HRV seemingly modulates the accessory function of mononuclear phagocytes and DC through different mechanisms.

Importantly, HRV14 effects were not detectable upon addition of the virus to the MLR cultures or due to a brief pretreatment of DC with HRV14 but required a co-culture of at least $24 \mathrm{~h}$. During this time, HRV14 obviously modulates the co-stimulatory repertoire of DC in a special way. The expression of most co-stimulatory molecules analyzed in this study was not affected on R-DC. For instance, CD40, CD58, CD80 and CD86 expression was similar on R-DC and on untreated DC. Likewise, expression of MHC class I and MHC class II molecules, a known target of viral escape mechanisms, was not downregulated on R-DC. Thus, the clearly reduced stimulatory capacity of R-DC did not correlate with a reduced ability to deliver signals 1 and 2 to T cells. Since we observed that R-DC gain a bystander inhibitory function, we concluded that HRV14 may alter the accessory phenotype by inducing inhibitory receptor(s) on DC.

We could indeed identify two of such receptors on RDC, which seem to deliver inhibitory signals to $T$ cells, B7-H1 and Sn. B7-H1, a recently described member of the B7 molecule family, is a well-defined accessory molecule with inhibitory effects through its receptor PD-1 on the T-cell side [62]. B7-H1 is critically involved in the induction and maintenance of T-cell anergy induced by IL-10treated DC [63]. Yet, blocking of B7-H1 was not sufficient to revert the deep anergic state in $\mathrm{T}$ cells triggered by RDC. Since other inhibitory accessory molecules on DC are not characterized so far, we had to search for such candidate structures on R-DC. By combining classical hybridoma technology and a potent retroviral expression cloning approach using a cDNA library from DC, we could identify $\mathrm{Sn}$ as an inhibitory accessory molecule on R-DC. Sn is a member of sialic acid binding lectin family of I-type lectins which preferentially binds to sialylated carbohydrate structures (e.g. NeuAca2,3-Gal) [64]. Sn is a frequently used marker for macrophages because it is not expressed on monocytes, lymphocytes and DC. Our finding that $\mathrm{Sn}$ is strongly induced on DC upon HRV14 treatment after 1-2 days correlates with the appearance of the inhibitory phenotype of R-DC. CD43 has been recently described as ligand for $\mathrm{Sn}$ on T cells [65]. How might this interaction contribute in the inhibition of $\mathrm{T}$ cell activation by R-DC? CD43 is known as an adhesion molecule involved in APC-T-cell interaction but is also known as barrier molecule. As such, CD43 is removed during APC-T-cell interaction from the immunological synapse to the distal pole of the $\mathrm{T}$ cell. This re-localization of CD43 is an active process dependent on reorganizations of the cytoskeleton. If an APC like our R-DC expresses a counter-receptor for CD43, it seems to be intriguing that this interaction might interfere with an appropriate movement of CD43 to the distal pole. As a consequence, synapse formation required for complete T-cell activation might be disturbed. Together with inhibitory signals arising from B7-H1-PD-1 interactions, this might explain the induction of a deep anergy in $\mathrm{T}$ cells upon R-DC activation.

The mechanism of B7-H1 and Sn induction in R-DC and the role of ICAM-1 are not yet understood. Signal transduction via ICAM-1 has been described but may not be sufficient. We observed that engagement and crosslinking of ICAM-1 with CD54 mAb RR1/1 did not trigger Sn expression on DC but inhibited HRV-induced Sn expression.

Interestingly, ICAM-1 is not only the receptor for rhinoviruses but also for other pathogens including Coxsackie viruses and Plasmodium falciparum [66]. For plasmodium-infected erythrocytes it has indeed been recently demonstrated that they inhibit DC maturation. Thus, ICAM-1 is not only an important adhesion molecule but like TLRs may also play an important role in sensing pathogens. However, our observations implicate that in contrast to TLRs, engagement of ICAM-1 on DC might trigger inhibitory signals which shut off the T-cell stimulatory function of DC. 
Fig. 1. Interaction of human rhinoviruses (HRV) with the mononuclear phagocyte cell system that subverts the T-cell stimulatory function of dendritic cells (DC) and induces IL-10 production in monocytes.

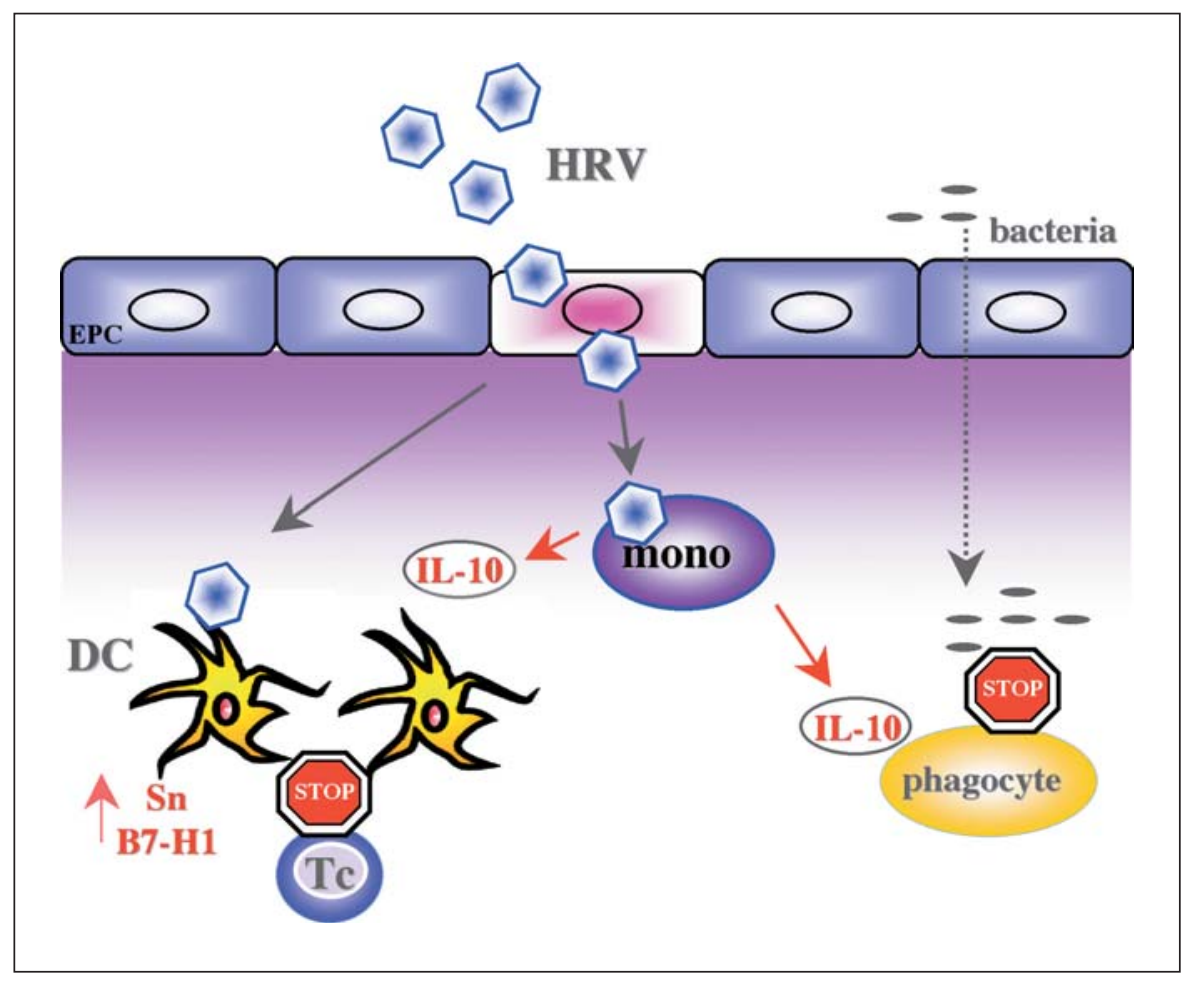

\section{Conclusion and Hypothesis}

On the basis of these observations it is tempting to speculate that the unique functional modulation of DC and monocytes by HRV might have adverse effects on local immunity in areas of HRV infection (fig. 1). Such reduced local immunocompetence may predispose affected individuals to secondary infections and could explain the frequently observed occurrence of sinusitis, otitis media, bronchitis, and pneumonia in HRV-infected persons.

HRV are highly specialized and only infect humans with all the well-known syndromes of a cold. HRV are probably the most successful human pathogens and it has been estimated that HRV colds per se will keep an average person in bed for about a whole year of their lives. Thus, HRV have to be real experts of the human immune system and apply efficient tricks to blunt antiviral immune responses. However, this is not necessarily a big concern. Cookson and Moffatt [67] have recently suggested that this may be part of our immune system's regular training, so HRV infections might be beneficial for us. It is amazing that up to $40 \%$ of HRV infections occur asymptomatically. The reasons for such a phenomenon are unclear. We hypothesize that the host/HRV relation- ship evolves towards a symbiotic relationship, which is beneficial for the virus and offers a natural (harmless) training partner for humans. Importantly, these training sessions may not only strengthen our immune system, but recent progress in the understanding of the immunomodulatory properties of HRV suggest that this may also be used to set the threshold of immune responses in the upper and maybe also lower respiratory tract. In persons with a certain predisposition, such as atopy, these 'training sessions' may have adverse effects and lead to asthma and maybe even autoimmunity such as multiple sclerosis. 


\section{References}

1 Alcami A, Koszinowski UH: Viral mechanisms of immune evasion. Trends Microbiol 2000;8:410-418.

-2 Finlay BB, McFadden G: Anti-immunology: evasion of the host immune system by bacterial and viral pathogens. Cell 2006;124:767782 .

3 McFadden G, Murphy PM: Host-related immunomodulators encoded by poxviruses and herpesviruses. Curr Opin Microbiol 2000;3:371-378.

$\checkmark 4$ Elena SF, Carrasco P, Daros JA, Sanjuan R: Mechanisms of genetic robustness in RNA viruses. EMBO Rep 2006;7:168-173.

5 Couch RB: Rhinoviruses, ed 3. New York, Raven Press, 1996.

-6 Pitkaranta A, Hayden FG: Rhinoviruses: important respiratory pathogens. Ann Med 1998;30:529-537.

7 Whitton JL, Cornell CT, Feuer R: Host and virus determinants of picornavirus pathogenesis and tropism. Nat Rev Microbiol 2005;3:765-776.

$>8$ Bella J, Rossmann MG: ICAM-1 receptors and cold viruses. Pharm Acta Helv 2000;74: 291-297.

-9 Rossmann MG, Bella J, Kolatkar PR, He Y, Wimmer E, Kuhn RJ, Baker TS: Cell recognition and entry by rhino- and enteroviruses. Virology 2000;269:239-247.

10 Rueckert RR: Picornaviridae: The Viruses and Their Replication; in Fields BN, Knipe DM, Howley PM (eds): Fields Virology, ed 2. Philadelphia, Lippincott-Raven, 1996, pp 477-522.

11 Heikkinen T, Jarvinen A: The common cold. Lancet 2003;361:51-59.

-12 Nurani G, Lindqvist B, Casasnovas JM: Receptor priming of major group human rhinoviruses for uncoating and entry at mild low pH environments. J Virol 2003;77: 11985-11991.

13 Xing L, Casasnovas JM, Cheng RH: Structural analysis of human rhinovirus complexed with ICAM-1 reveals the dynamics of receptor-mediated virus uncoating. J Virol 2003;77:6101-6107.

14 Petersen JF, Cherney MM, Liebig HD, Skern T, Kuechler E, James MN: The structure of the 2 a proteinase from a common cold virus: a proteinase responsible for the shut-off of host-cell protein synthesis. EMBO J 1999;18: 5463-5475.

-15 Einarsson O, Geba GP, Zhu Z, Landry M, Elias JA: Interleukin-11: stimulation in vivo and in vitro by respiratory viruses and induction of airways hyperresponsiveness. J Clin Invest 1996;97:915-924.

-16 Van Cauwenberge PB, van Kempen MJ, Bachert C: The common cold at the turn of the millennium. Am J Rhinol 2000;14:339343.
17 Psarras S, Volonaki E, Skevaki CL, Xatzip salti M, Bossios A, Pratsinis H, Tsigkos S, Gourgiotis D, Constantopoulos AG, Papapetropoulos A, Saxoni-Papageorgiou $\mathrm{P}$, Papadopoulos NG: Vascular endothelial growth factor-mediated induction of angiogenesis by human rhinoviruses. J Allergy Clin Immunol 2006;117:291-297.

18 Schroth MK, Grimm E, Frindt P, Galagan DM, Konno SI, Love R, Gern JE: Rhinovirus replication causes Rantes production in primary bronchial epithelial cells. Am J Respir Cell Mol Biol 1999;20:1220-1228.

19 Spurrell JC, Wiehler S, Zaheer RS, Sanders SP, Proud D: Human airway epithelial cells produce IP-10 (CXCL10) in vitro and in vivo upon rhinovirus infection. Am J Physiol 2005;289:L85-L95.

20 Message SD, Johnston SL: Host defense function of the airway epithelium in health and disease: clinical background. J Leukoc Biol 2004;75:5-17.

21 Teran LM, Johnston SL, Schroder JM, Church MK, Holgate ST: Role of nasal interleukin-8 in neutrophil recruitment and activation in children with virus-induced asthma. Am J Respir Crit Care Med 1997;155: 1362-1366.

22 Fahy JV, Kim KW, Liu J, Boushey HA: Prominent neutrophilic inflammation in sputum from subjects with asthma exacerbation. J Allergy Clin Immunol 1995;95:843-852.

23 Gleich GJ: Mechanisms of eosinophil-associated inflammation. J Allergy Clin Immunol 2000;105:651-663.

24 Katze MG, He Y, Gale M Jr: Viruses and interferon: a fight for supremacy. Nat Rev Immunol 2002;2:675-687.

25 Wark PA, Johnston SL, Bucchieri F, Powell R, Puddicombe S, Laza-Stanca V, Holgate ST, Davies DE: Asthmatic bronchial epithelial cells have a deficient innate immune response to infection with rhinovirus. J Exp Med 2005;201:937-947.

26 Kainulainen L, Varpula M, Liippo K, Svedstrom E, Nikoskelainen J, Ruuskanen O: Pulmonary abnormalities in patients with primary hypogammaglobulinemia. J Allergy Clin Immunol 1999;104:1031-1036.

27 Gern JE, Dick EC, Kelly EA, Vrtis R, Klein B: Rhinovirus-specific $\mathrm{T}$ cells recognize both shared and serotype-restricted viral epitopes. J Infect Dis 1997;175:1108-1114.

28 Wimalasundera SS, Katz DR, Chain BM: Characterization of the $\mathrm{T}$-cell response to human rhinovirus in children: implications for understanding the immunopathology of the common cold. J Infect Dis 1997;176:755759.

29 Handzel ZT, Busse WW, Sedgwick JB, Vrtis R, Lee WM, Kelly EA, Gern JE: Eosinophils bind rhinovirus and activate virus-specific $\mathrm{T}$ cells. J Immunol 1998;160:1279-1284.
30 Kriesel JD, Sibley WA: The case for rhinoviruses in the pathogenesis of multiple sclerosis. Mult Scler 2005;11:1-4.

31 Mallia P, Johnston SL: Mechanisms and experimental models of chronic obstructive pulmonary disease exacerbations. Proc Am Thorac Soc 2005;2:361-366, 371-362.

32 Murray CS, Simpson A, Custovic A: Allergens, viruses, and asthma exacerbations. Proc Am Thorac Soc 2004;1:99-104.

33 Nicholson KG, Kent J, Ireland DC: Respiratory viruses and exacerbations of asthma in adults. BMJ 1993;307:982-986.

34 Pitkaranta A, Hayden FG: Respiratory viruses and acute otitis media. N Engl J Med 1999; 340:2001-2002.

-35 Gern JE, Dick EC, Lee WM, Murray S, Meyer K, Handzel ZT, Busse WW: Rhinovirus enters but does not replicate inside monocytes and airway macrophages. J Immunol 1996; 156:621-627.

>36 Stockl J, Vetr H, Majdic O, Zlabinger G, Kuechler E, Knapp W: Human major group rhinoviruses downmodulate the accessory function of monocytes by inducing IL-10. J Clin Invest 1999;104:957-965.

37 Haller O, Kochs G: Interferon-induced Mx proteins: dynamin-like GTPases with antiviral activity. Traffic 2002;3:710-717.

38 Makela MJ, Halminen M, Ruuskanen O, Puhakka T, Pirhonen J, Julkunen I, Ilonen J: Lack of induction by rhinoviruses of systemic type I interferon production or enhanced MxA protein expression during the common cold. Eur J Clin Microbiol Infect Dis 1999;18: 665-668.

39 Peng T, Kotla S, Bumgarner RE, Gustin KE: Human rhinovirus attenuates the type I interferon response by disrupting activation of interferon regulatory factor 3. J Virol 2006; 80:5021-5031.

40 Springer TA: Leucocyte adhesion to cells. Scand J Immunol 1990;32:211-216.

-41 Sligh JE Jr, Ballantyne CM, Rich SS, Hawkins HK, Smith CW, Bradley A, Beaudet AL: Inflammatory and immune responses are impaired in mice deficient in intercellular adhesion molecule-1. Proc Natl Acad Sci USA 1993;90:8529-8533.

42 Gaglia JL, Greenfield EA, Mattoo A, Sharpe $\mathrm{AH}$, Freeman GJ, Kuchroo VK: Intercellular adhesion molecule-1 is critical for activation of CD28-deficient T cells. J Immunol 2000; 165:6091-6098.

43 Smith TJ, Chase ES, Schmidt TJ, Olson NH, Baker TS: Neutralizing antibody to human rhinovirus 14 penetrates the receptor-binding canyon. Nature 1996;383:350-354.

44 Piela-Smith TH, Aneiro L, Korn JH: Binding of human rhinovirus and T cells to intercellular adhesion molecule-1 on human fibroblasts. Discordance between effects of IL-1 and IFN- $\gamma$. J Immunol 1991;147:1831-1836. 
-45 Gern JE, Joseph B, Galagan DM, Borcherding WR, Dick EC: Rhinovirus inhibits antigen-specific $\mathrm{T}$-cell proliferation through an intercellular adhesion molecule-1-dependent mechanism. J Infect Dis 1996;174:11431150.

-46 Durieu-Trautmann O, Chaverot N, Cazaubon S, Strosberg AD, Couraud PO: Intercellular adhesion molecule-1 activation induces tyrosine phosphorylation of the cytoskeleton-associated protein cortactin in brain $\mathrm{mi}$ crovessel endothelial cells. J Biol Chem 1994; 269:12536-12540.

-47 Etienne-Manneville S, Chaverot N, Strosberg AD, Couraud PO: ICAM-1-coupled signaling pathways in astrocytes converge to cyclic AMP response element-binding protein phosphorylation and TNF- $\alpha$ secretion. J Immunol 1999;163:668-674.

48 Holland J, Owens T: Signaling through intercellular adhesion molecule-1 (ICAM-1) in a B-cell lymphoma line. The activation of Lyn tyrosine kinase and the mitogen-activated protein kinase pathway. J Biol Chem 1997; 272:9108-9112.

49 Casasnovas JM: The dynamics of receptor recognition by human rhinoviruses. Trends Microbiol 2000;8:251-254.

50 Kirchberger S, Vetr H, Majdic O, Stockinger $\mathrm{H}$, Stöckl J: Engagement of ICAM-1 by major group rhinoviruses activates the LFA-1/ ICAM-3 cell adhesion pathway in mononuclear phagocytes. Immunobiology 2006; 211:537-547.

51 Goerdt S, Kodelja V, Schmuth M, Orfanos CE, Sorg C: The mononuclear phagocytedendritic cell dichotomy: myths, facts, and a revised concept. Clin Exp Immunol 1996; 105:1-9.
52 Peters JH, Gieseler R, Thiele B, Steinbach F: Dendritic cells: from ontogenetic orphans to myelomonocytic descendants. Immunol Today 1996;17:273-278.

53 De Waal Malefyt R, Haanen J, Spits H, Roncarolo MG, te Velde A, Figdor C, Johnson K, Kastelein R, Yssel H, de Vries JE: Interleukin-10 (IL-10) and viral IL-10 strongly reduce antigen-specific human T-cell proliferation by diminishing the antigen-presenting capacity of monocytes via downregulation of class II major histocompatibility complex expression. J Exp Med 1991;174: 915-924.

54 Karp CL, Wysocka M, Wahl LM, Ahearn JM, Cuomo PJ, Sherry B, Trinchieri G, Griffin DE: Mechanism of suppression of cell-mediated immunity by measles virus. Science 1996;273:228-231.

55 Papadopoulos NG, Stanciu LA, Papi A, Holgate ST, Johnston SL: Rhinovirus-induced alterations on peripheral blood mononuclear cell phenotype and costimulatory molecule expression in normal and atopic asthmatic subjects. Clin Exp Allergy 2002;32: 537-542.

56 Howard M, O’Garra A: Biological properties of interleukin-10. Immunol Today 1992;13: 198-200.

57 Gerard C, Bruyns C, Marchant A, Abramowicz D, Vandenabeele P, Delvaux A, Fiers W, Goldman M, Velu T: Interleukin-10 reduces the release of tumor necrosis factor and prevents lethality in experimental endotoxemia. J Exp Med 1993;177:547-550.

58 Kuhn R, Lohler J, Rennick D, Rajewsky K, Muller W: Interleukin-10-deficient mice develop chronic enterocolitis. Cell 1993;75: 263-274.

59 Grissell TV, Powell H, Shafren DR, Boyle MJ Hensley MJ, Jones PD, Whitehead BF, Gibson PG: Interleukin-10 gene expression in acute virus-induced asthma. Am J Respir Crit Care Med 2005;172:433-439.
60 Palucka K, Banchereau J: How dendritic cells and microbes interact to elicit or subvert protective immune responses. Curr Opin Immunol 2002;14:420-431.

61 Kirchberger S, Majdic O, Steinberger P, Bluml S, Pfistershammer K, Zlabinger G, Deszcz L, Kuechler E, Knapp W, Stockl J: Human rhinoviruses inhibit the accessory function of dendritic cells by inducing sialoadhesin and B7-H1 expression. J Immunol 2005;175:1145-1152.

62 Greenwald RJ, Freeman GJ, Sharpe AH: The B7 family revisited. Annu Rev Immunol 2005;23:515-548.

63 Selenko-Gebauer N, Majdic O, Szekeres A, Hofler G, Guthann E, Korthauer U, Zlabinger G, Steinberger P, Pickl WF, Stockinger H, Knapp W, Stockl J: B7-H1 (programmed death-1 ligand) on dendritic cells is involved in the induction and maintenance of T-cell anergy. J Immunol 2003;170:3637-3644.

64 Hartnell A, Steel J, Turley H, Jones M, Jackson DG, Crocker PR: Characterization of human sialoadhesin, a sialic acid binding receptor expressed by resident and inflammatory macrophage populations. Blood 2001;97:288-296.

65 Van den Berg TK, Nath D, Ziltener HJ, Vestweber D, Fukuda M, van Die I, Crocker PR: Cutting edge: CD43 functions as a T-cell counter-receptor for the macrophage adhesion receptor sialoadhesin (Siglec-1). J Immunol 2001;166:3637-3640.

66 Van de Stolpe A, van der Saag PT: Intercellular adhesion molecule-1. J Mol Med 1996; 74:13-33.

67 Cookson WO, Moffatt MF: Asthma: an epidemic in the absence of infection? Science 1997;275:41-42. 